\title{
Considering on return stroke current wave shape for tall objective cases
}

\begin{abstract}
The return stroke current along the lightning channel for striking of the lightning to tall objective case is different from ground surface case with many factors affect this phenomenon. This study considers the engineering related to return stroke current models where the general equations are expressed and different models are generated. The results obtained will be compared and discussed.
\end{abstract}

Keyword: Lightning channel; Return stroke current; Tall object 